\author{
Riann M. Palmieri \\ Arthur Weltman \\ Jeffrey E. Edwards \\ James A. Tom \\ Ethan N. Saliba \\ Danny J. Mistry \\ Christopher D. Ingersoll
}

Received: 26 February 2004

Accepted: 12 May 2004

Published online: 1 February 2005

(C) Springer-Verlag 2005

\section{R. M. Palmieri ( $\square)$}

Neuromuscular Research Laboratory,

Division of Kinesiology,

University of Michigan,

401 Washtenaw Avenue,

Ann Arbor, MI 48109, USA

E-mail: riannp@umich.edu

Tel.: + 1-734-6153154

Fax: + 1-734-9361925

\section{A. Weltman}

Exercise Physiology Laboratory, Department of Human Services,

University of Virginia, PO Box 400407,

210 Emmet Street, South,

Charlottesville, VA 22904-4407, USA

\section{J. E. Edwards}

Human Performance Laboratory, Department of Physical Education, Indiana State University, Terre Haute, IN 47809, USA

\section{J. A. Tom}

Orthopedic Surgery Control,

Drexel University Health Sciences,

New College Building, 245 N 15th St,

Philadelphia, PA, USA

E. N. Saliba - D. J. Mistry · C. D. Ingersoll Exercise and Sports Injury Laboratory, Department of Human Services,

University of Virginia, PO Box 400407,

210 Emmet Street, South,

Charlottesville, VA 22904-4407, USA

\title{
Pre-synaptic modulation of quadriceps arthrogenic muscle inhibition
}

Abstract Arthrogenic muscle inhibition (AMI) impedes rehabilitation following knee joint injury by preventing activation of the quadriceps. AMI has been attributed to neuronal reflex activity in which altered afferent input originating from the injured joint results in a diminished efferent motor drive to the quadriceps muscles. Beginning to understand the mechanisms responsible for muscle inhibition following joint injury is vital to control or eliminate this phenomenon. Therefore, the purpose of this investigation is to determine if quadriceps AMI is mediated by a presynaptic regulatory mechanism. Eight adults participated in two sessions: in one session their knee was injected with saline and in the other session it was not. The maximum Hoffmann reflex (H-reflex), M-wave, reflex activation history, plasma epinephrine, and norepinephrine were recorded at: baseline, post needle stick, post lidocaine, and 25 and $45 \mathrm{~min}$ post effusion. Measures for the control condition were matched to the effusion condition. The percent of the unconditioned reflex amplitude for reflex activation history and the maximum H-reflex were decreased at 25 and 45 min post effusion as compared to measures taken at baseline, post needle stick, and post lidocaine $(P<0.05)$. No differences were noted for the maximum Mwave or plasma epinephrine and norepinephrine levels in either the effusion or noneffusion admission $(P>0.05)$. No differences were detected at any time interval for any measure during the control admission $(P>0.05)$. Quadriceps AMI elicited via an experimental knee joint effusion is, at least in part, mediated by a presynaptic mechanism.

Keywords Weakness - Quadriceps · Activation failure $\cdot$ Spinal reflex . Catecholamines 


\section{Introduction}

Knee joint pathology, whether acute [12, 24], chronic $[14,15]$ or experimentally induced $[8,20,25]$, results in weakness of the quadriceps musculature acting about the knee joint complex. This phenomenon has been termed arthrogenic muscle inhibition (AMI) and is defined as an ongoing reflex inhibition of musculature surrounding a joint following distension or damage to structures of that joint [6]. AMI hinders rehabilitation by preventing gains in strength [11-13] and restoration of normal proprioceptive function [11], resulting in persistent functional deficiencies [12, 15], the possibility of reinjury [26], and potentially placing patients at risk for chronic degenerative joint conditions [27]. In order to develop an effective intervention to reduce or remove AMI, thereby improving the chances for successful rehabilitation of the injured joint, it is imperative to understand the neuromuscular mechanisms responsible for the quadriceps weakness.

The neuromuscular system is an intricate network operating to regulate motor output and to control body movement. Interneurons are key components in the spinal circuitry, accounting for the majority of all neurons in the spinal cord, and they function to transmit excitatory and inhibitory signals to other interneurons as well as to alpha and gamma motoneurons [1]. Although a reduction in quadriceps alpha motoneuron output is ultimately responsible for AMI, the interneurons involved in regulating the excitability of the quadriceps motoneuron pool remain unknown. AMI has been hypothesized to involve interneurons controlling presynaptic mechanisms [6] Reflex activation history, a presynaptic regulatory mechanism, can be estimated using a modified Hoffmann reflex (H-reflex) protocol [29].

Previous activation of the Ia-motoneuron synapse results in decreased neurotransmitter release and inhibition of the reflex pathway [3]. The depression associated with reflex activation is probably related with other presynaptic mechanisms functioning to regulate the gain of motoneuron output [29]. Modulation of reflex depression associated with the frequency of reflex activation may reduce the efficacy of afferent transmission to the motoneuron pool, thereby decreasing efferent output to the quadriceps musculature following knee joint effusion. The ability to gate motoneuron activity in an injured state could allow for precise control of motor function. Uncontrolled, painful knee extensor moments could be prevented by reducing excitatory input to the quadriceps.

Knee joint injury is known to result in quadriceps inhibition, yet the neurophysiological mechanisms behind these changes remain unknown. Presynaptic pathways may be involved in AMI following joint injury. Therefore, the purpose of this investigation was to examine a presynaptic regulatory mechanism responsible for gating motoneuron pool output before and after the induction of an experimental knee joint effusion. Specifically, reflex activation history was examined in the vastus medialis (VM) to determine its role, if any, in the neuromuscular excitability changes seen following the joint injury.

\section{Methods}

A $2 \times 5$ factorial design was used in this study. The two independent variables were condition (effusion/noneffusion) and measurement interval (baseline, post needle stick, post lidocaine, 25 min post effusion, and $45 \mathrm{~min}$ post effusion). The dependent variables were Hmax, Mmax, and percent of the unconditioned reflex amplitude for paired reflex depression (PRD), as well as plasma epinephrine and norepinephrine levels.

\section{Subjects}

Eight healthy subjects (three females and five males; age $24 \pm 12$ years, height $167.64 \pm 7.31 \mathrm{~cm}$, weight $78.24 \pm 14.74 \mathrm{~kg}$ ) with no previous lower-extremity injury resulting in surgery, no injury to the lower extremity requiring treatment in the previous 2 years, and a measurable quadriceps H-reflex and Muscle response (Mresponse) were recruited to volunteer for this study. The volunteers had no history of an adverse reaction to Xylocaine, neurological conditions, or blood disorders. The subjects were not currently taking any medication that could affect the central nervous system function (i.e., antidepressants, barbiturates, narcotics, and sedatives). Additionally, the female subjects were not taking oral contraceptives. All females who qualified for this study reported for testing during the early follicular phase of their menstrual cycle. The subjects were assessed for inclusion criteria via a preparticipation history questionnaire, a physical examination, and a reflex screening. Informed consent to participate in this study was obtained prior to subject participation. Human subject approval (no. 10322) was obtained from the Human Investigations Committee prior to beginning the study.

\section{Instrumentation}

Hoffmann reflex and M-response measurements were collected using surface electromyography (MP150, BIOPAC Systems Inc., Santa Barbara, CA, USA). Signals were amplified (EMG100C, BIOPAC Systems Inc.; Gain 1000) from disposable, $10 \mathrm{~mm}$ pregelled 
Ag-AgCl electrodes (EL503, BIOPAC Systems Inc.). The EMG signal was band-pass filtered from 10 to $500 \mathrm{~Hz}$ and sampled at $1024 \mathrm{~Hz}$ with a common-mode rejection ratio of $110 \mathrm{~dB}$. Reflex measurements were elicited using the BIOPAC stimulator module (STM100A, BIOPAC Systems Inc.) with a 200-V (maximum) stimulus isolation adapter (STMISOC, BIOPAC Systems Inc.), $2 \mathrm{~mm}$ shielded disc electrode (EL254S, BIOPAC Systems Inc.), and a $7 \mathrm{~cm}$ dispersive pad.

\section{Reflex screening}

A screening session was held for all the volunteers prior to enrollment in the study. A general explanation of the study and its significance was given along with an explanation of the measurement protocol and the risks involved in participating, and informed consent was obtained. H-reflex and M-response measurements were recorded in the VM to ensure that the subjects had readable measurements necessary for data collection. If readable measurements were not obtained, the subject was dismissed (six subjects had to be dismissed because of an unreadable H-reflex or because Mmax couldn't be obtained). If an H-reflex and M-response were elicited, the risks involved in participation were explained, informed consent was obtained, and volunteers were randomly assigned to a testing order (effusion/noneffusion). All eight subjects completed both the testing conditions (effusion/noneffusion). Testing conditions were separated by at least 3 days. Each subject was considered as a block, and the sequence of the admissions was assigned to each subject by way of a computergenerated random permutation.

\section{Subject preparation}

Each subject was shaved, debrided, and cleaned with isopropyl alcohol for reception of the EMG electrodes. Two surface EMG electrodes were placed over the muscle belly of the VM and a ground electrode was placed on the medial malleolus. Subjects had a cathode placed over the femoral nerve and an anode positioned on the gluteus. Adhesive collars were applied to the cathodes in order to maintain their position over the nerves for the duration of the data collection.

\section{Testing procedures}

\section{Effusion testing sessions}

The volunteers were admitted into the General Clinical Research Center (GCRC) the evening prior to testing and had an indwelling venous catheter placed in their nondominant forearm at 2200 hours. The subjects were shaved over the sites for EMG electrode placement. The subjects were fed a standardized snack (noncaffeinated) at 2300 hours (they then fasted until the completion of the testing protocol). They were then asked to prepare for bed and lights were turned off by 2400 hours. At 0550 hours the subjects were awoken and asked to void. They were then asked to return to bed and remain lying down until completion of the test. Beginning at 0630 hours, baseline neurophysiological measurements (Hmax, Mmax, $\mathrm{H}$ at $\sim 15 \%$ of Mmax, and the test $\mathrm{H}$ reflex) were recorded. Immediately following these measurements, a blood sample was withdrawn on ice and stored for catecholamine analysis. The volunteers were now prepared for the knee injection. A $25 \mathrm{G} 1.5$ " needle was inserted and removed to mimic the puncture caused during the Xylocaine injection. The Xylocaine injection was performed followed by the injection of the sterile saline. Neurophysiological measurements and blood samples were recorded after the first needle insertion and after the Xylocaine injections. Measurements and blood samples were taken again at 25 and 45 min post saline injection. Upon completion of testing, the catheter and electrodes were removed; the subjects were fed and then discharged from the GCRC.

\section{Noneffusion testing sessions}

Subject admission to the GCRC was the same as described above. All the measurements and blood samples were time-matched to the effusion condition. No injections were administered during this admission.

\section{$\mathrm{H}$-reflex and M-response procedures}

The volunteers were positioned supine with the involved knee in approximately $15^{\circ}$ of flexion. The subjects were asked to place their hands to their sides with their palms facing up, and to keep their head facing forward [7, 10].

A series of 1-ms [21] square wave pulses were delivered to the volunteers in order to obtain the peak-topeak amplitude of the maximum H-reflex (Hmax) and maximum M-response (Mmax). The stimuli were given with 10-s rest intervals in between to ensure that postactivation depression did not interfere with the H-reflex amplitude [23]. Lower-intensity stimuli were used to depolarize Ia afferents and elicit an H-reflex, while higher-intensity stimuli were necessary to depolarize the motor axons resulting in an M-response. Prior to stimulus delivery, $30 \mathrm{~ms}$ of background EMG activity was recorded. When the Hmax and Mmax were found, five measurements were elicited and recorded. The stimulus intensity was then adjusted to record an H-reflex at $\sim 15 \%$ of the Mmax, and five measurements were recorded at this constant stimulation intensity. 


\section{PRD procedures}

The method for eliciting PRD (a measure of reflex activation history) in the soleus of humans has been previously described [29]. The same protocol as that described for the soleus was used in the quadriceps. Dual stimuli were delivered to the femoral nerve $80 \mathrm{~ms}$ apart to evoke quadriceps $\mathrm{H}$-reflexes. Stimulus intensity was set to elicit H-reflexes at $\sim 15 \%$ of Mmax. The depression of the second H-reflex relative to the first $(\%$ depression) was used. The decrease in the second reflex is referred to as the PRD, and represents modulation of processes controlling rate-dependent reflex depression and the influence of the reflex activation history (Fig. 1) [29]. Eight measures were recorded, averaged, and used for data analysis.

\section{Joint effusion procedures}

An area superolateral to the patella was cleaned with alcohol and povidone-iodine. The patient's lower limb was extended while lying supine in bed. Using a 25gauge needle a needle stick was made subcutaneously. This injection was necessary to evaluate the effect of a needle stick on our dependent measures. Using a sterile, disposable syringe with a $25 \mathrm{G} 1.5$ " needle, $3 \mathrm{ml}$ of $1 \%$ Xylocaine was injected subcutaneously for anesthetic purposes. When injecting the saline into the intraarticular space, the physician manually everted and moved the patella laterally using his free hand. Using a disposable syringe with a $21 \mathrm{G}$ needle attached, $60 \mathrm{ml}$ of saline was injected. The needle was advanced between the articular surface of the patellofemoral joint at the midpoint of the patella [17]. Sterile disposable gloves were worn during each injection for all the subjects.

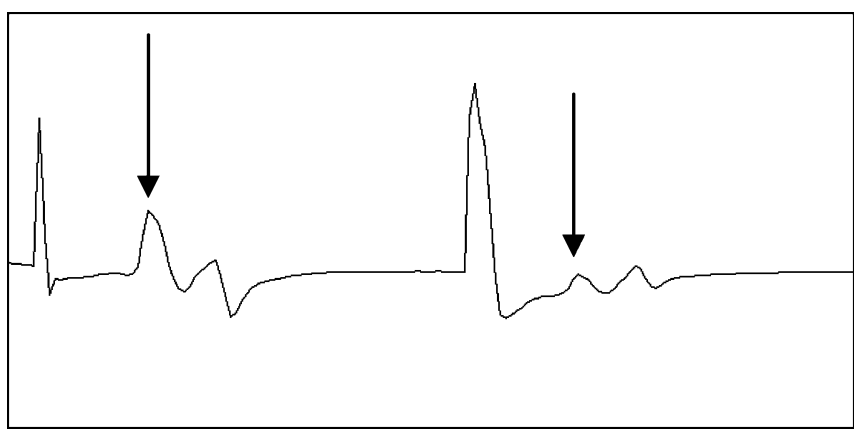

Fig. 1 A PRD tracing in the VM from a single subject. The amplitude of the second reflex is depressed compared to the first. The latency for both responses is $18.55 \mathrm{~ms}$
Catecholamine analysis

Catecholamine analysis was performed by Nichols Institute (Quest Diagnostics, Teterboro, NJ). The blood samples $(10 \mathrm{ml})$ were collected in prechilled vacutainer tubes. The plasma was separated in a refrigerated centrifuge within $30 \mathrm{~min}$ of collection and then frozen immediately at $-20^{\circ} \mathrm{C}$ in plastic vials.

The determination of plasma epinephrine and norepinephrine were performed using high-pressure liquid chromatography (HPLC) with electrochemical detection. This assay involves extraction of plasma with alumina, followed by HPLC. Prior to chromatography, an internal standard (IS) was added to each volunteer's sample and to tubes containing standards of known concentration. Fractionated catecholamine levels were determined by comparing the ratio of peak heights of patient samples to IS with the ratios of known standards to IS.

\section{Statistical analysis}

A mixed effects model for repeated measures was used for each dependent variable. The model specifications included two independent variables: admission and measurement interval. Admission was modeled as the between-subject factor, while measurement interval was modeled as a within-subject factor. Measurement interval by admission interaction was also considered. The model parameters were estimated by restricted maximum likelihood. The covariance matrix was modeled in a spatial power forum. The comparisons of interactions were formulated by one degree of freedom contrasts between the means for the groups. Multiple-comparison Type I error rate adjustment was based on a Fisher's least significant difference (FLSD) criterion with a Type I error rate of 0.05 .

Pearson product moment correlations were run between epinephrine and norepinephrine plasma concentration and Hmax at measurement intervals 25 and 45 min post effusion for the effusion admission. Correlations were considered significant at the 0.05 level. SAS version 8.0 (SAS Institute, Cary, NC) was used for all statistical analyses.

\section{Results}

\section{Hmax and Mmax}

An overall interaction was found between the measurement interval and admission for the H-reflex $\left(F_{4,56}=13.48 ; P<0.0001\right)$. Hmax during the effusion admission was greater at baseline than at $25 \mathrm{~min}$ $(P<0.0001)$ and $45 \mathrm{~min}(P<0.0001)$ post knee joint 


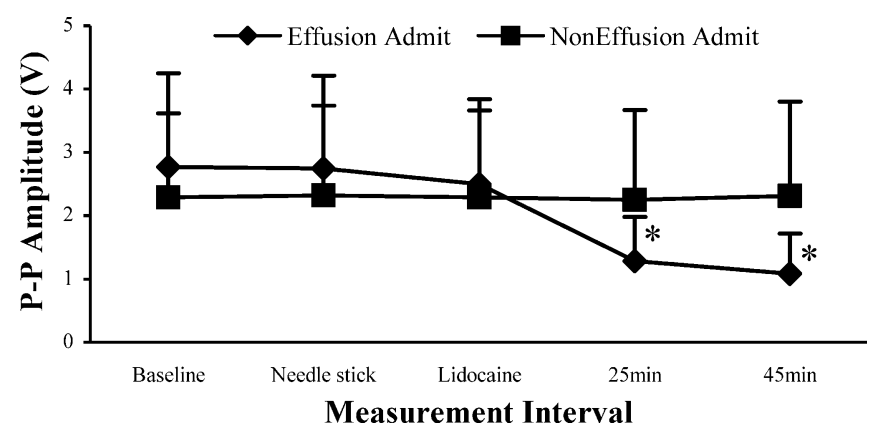

Fig. 2 Hmax amplitude at each measurement interval for the effusion and noneffusion admissions. Asterisks denote a significant difference from baseline

effusion (Fig. 2). Hmax amplitude was greater at 25 and 45 min post effusion than at post needle stick and post lidocaine $(P<0.0001)$. No difference was noted between Hmax at baseline and Hmax post needle stick $(P=0.91)$ or post lidocaine $(P=0.22)$. Hmax post needle stick did not differ from Hmax post lidocaine $(P=0.59)$ for the effusion admission. No differences in the Hmax were noted during the control admission at any measurement interval $(P>0.05)$

The omnibus test for Mmax failed to reveal a significant effect for admission $\left(F_{1,7}=0.69 ; P=0.4324\right)$, measurement interval $\left(F_{4,56}=3.25 ; P=0.20\right)$ (Fig. 3$)$ or measurement interval*admission $\left(F_{4,56}=1.32 ; P=0.27\right)$.

\section{Paired reflex depression}

A significant measurement interval by admission interaction was found $\left(F_{4,56}=12.96 ; P<0.0001\right)$. The conditioned reflex during the effusion admission decreased from baseline at $25 \mathrm{~min}(P<0.0001)$ and $45 \mathrm{~min}$ $(P<0.0001)$ post knee joint effusion (Fig. 4). The con-

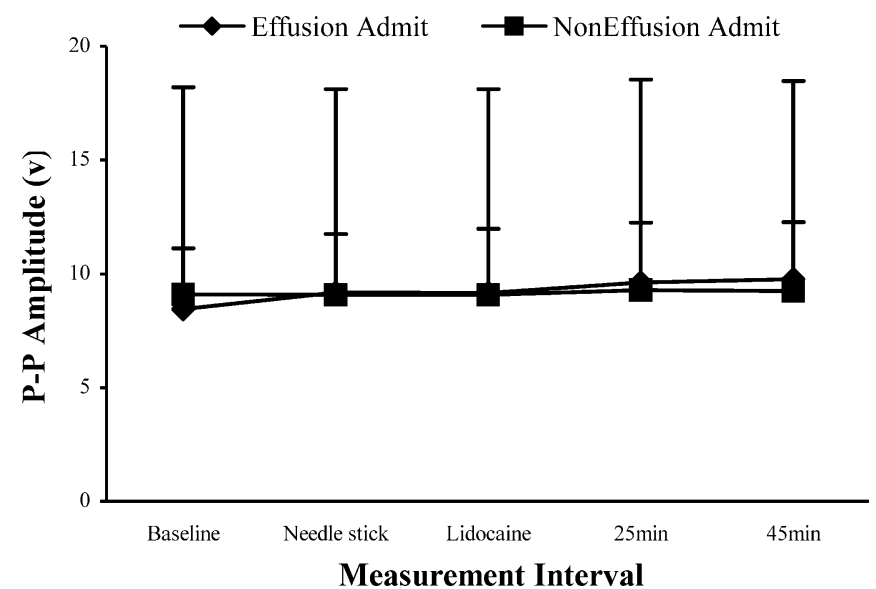

Fig. 3 Mmax amplitude at each measurement interval for the effusion and control admissions

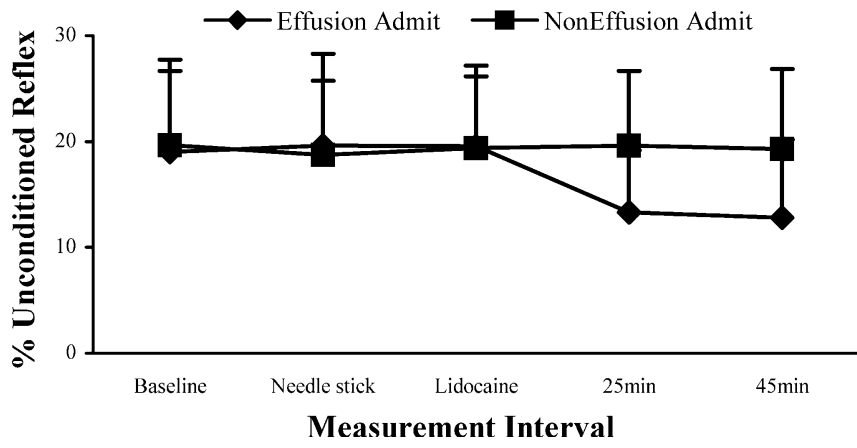

Fig. 4 Conditioned quadriceps H-reflex amplitude as a percentage of the unconditioned reflex amplitude for PRD

ditioned reflex post needle stick $(P=0.54)$ and post lidocaine $(P=0.58)$ did not change from baseline. No changes were noted at any measurement interval during the control admission $(P>0.05)$.

\section{Catecholamines}

In instances where catecholamine data points were missing, SAS used the harmonic means to analyze the data. Data points were missing for one subject at measurement intervals 25 and 45 min post effusion and for two subjects at the 45 min post effusion. Measurement of all these data points was during the noneffusion admission. The reason for the missing data was either an error in the analysis or difficulty with the drawing of blood. No differences in plasma epinephrine levels were noted for measurement interval $\left(F_{4,52.9}=1.56 ; P=0.21\right)$, admission $\left(F_{1,4}=1.61 ; P=0.22\right)$, or measurement interval*admission $\left(F_{4,52.9}=0.93 ; P=0.45\right)$ (Fig. 5$)$. Likewise, no differences were noted for plasma norepinephrine levels when compared for measurement interval $\left(F_{4,52.2}=2.58\right.$;

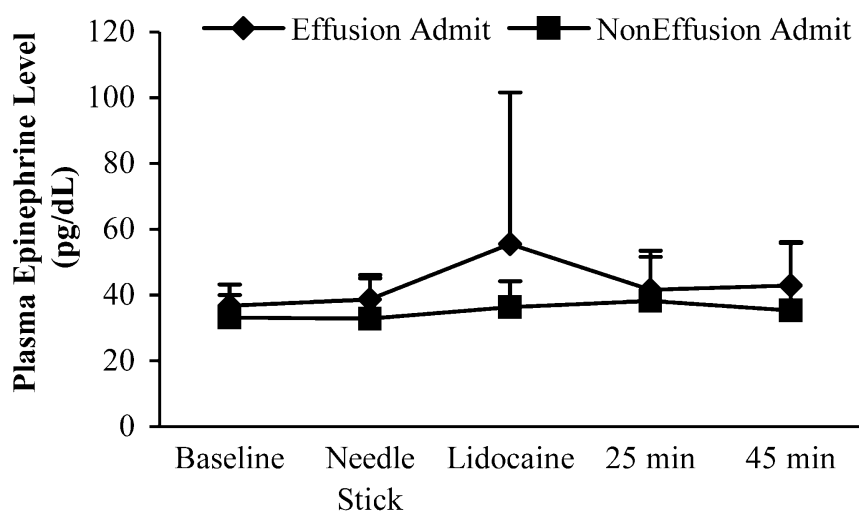

Measurement Intervals

Fig. 5 Plasma epinephrine levels at each measurement interval for the effusion and control admission 


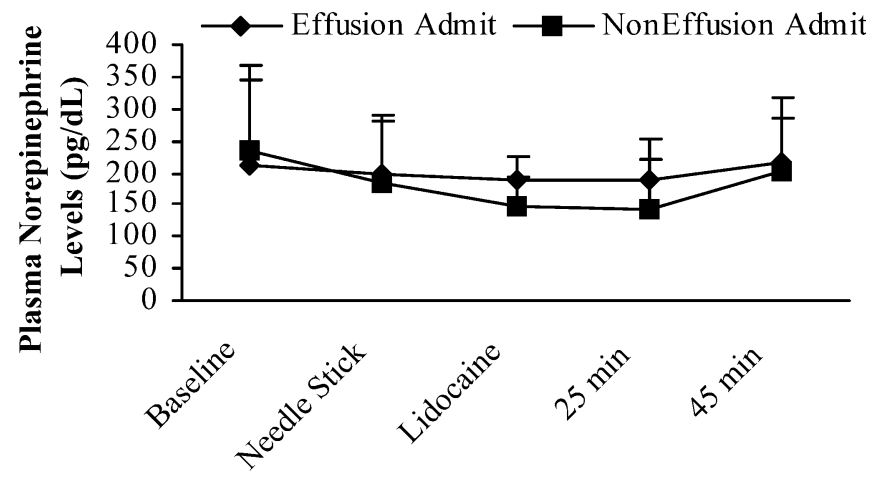

Measurement Intervals

Fig. 6 Plasma norepinephrine levels at each measurement interval for the effusion and control admission

$P=0.08)$, admission $\left(F_{1,6.5}=0.63 ; P=0.45\right)$, or measurement interval*admission $\left(F_{4,52.2}=0.74 ; \quad P=0.57\right)$ (Fig. 6).

\section{Correlations}

No relationship existed between plasma epinephrine and the quadriceps H-reflex at $25 \min (r=0.45 ; P=0.30)$ or 45 min $(r=0.54 ; P=0.15)$ post effusion. Likewise, no relationship was established between plasma norepinephrine and the quadriceps H-reflex at $25 \min (r=0.49$; $P=0.30)$ or $45 \min (r=-0.08 ; P=0.84)$ post effusion.

\section{Discussion}

Knee effusion results in quadriceps AMI [8, 9, 16, 20]. Our data suggests that AMI is due, in part, to a presynaptic spinal mechanism. Potentially, GABA-ergic interneurons responsible for classic presynaptic inhibition may contribute to the inhibition of the quadriceps musculature following injury. Our observations diverge from previous hypotheses that AMI is caused only by the activation of the Ib interneuron $[8,16]$.

Ruffini endings are stimulated when an effusion is present [5], alerting and activating central structures regarding the current state of the knee joint. Joint afferents are known to synapse on Ib interneurons [18], which has led researchers to hypothesize that the $\mathrm{Ib}$ inhibitory interneuron may contribute to quadriceps AMI. Little definitive evidence exists pointing to this interneuron being entirely responsible for the inhibition seen in the quadriceps musculature. Nonreciprocal inhibition mediated by Ib interneurons has been shown to play a role in eliciting the quadriceps inhibition that exists in the presence of a knee joint effusion [16]. During a quadriceps contraction nonreciprocal inhibition was not present, but when the knee was effused and the subjects contracted, nonreciprocal inhibition increased to approximately $17 \%$, providing some evidence to support the involvement of the Ib interneuron in AMI. Our data make it apparent that other mechanisms regulating muscle output are activated and contribute to quadriceps AMI when an effusion is present.

Reflex depression was increased following induction of the experimental effusion, leading us to speculate that a presynaptic regulatory control contributed to the decreased excitability of quadriceps motoneurons. The exact mechanism responsible for the rate-dependent modulation of the H-reflex remains unknown. Descending pathways are known to influence reflex activation history through spinal interneurons. Specifically, GABA-ergic interneurons responsible for presynaptic inhibition are influenced by descending controls $[19,28]$. Post-activation depression, and previous activation of a muscle, resulting in a decrease in the $\mathrm{H}$-reflex, can be viewed as being similar to the PRD protocol used in this study - both are the result of previous activation of a muscle. The amplitude of the H-reflex has been found to return to $66 \%$ of its original amplitude within 400-500 ms, which corresponds with the time course for classic pre-synaptic inhibition [4] and has led investigators to speculate about its involvement in post-activation depression [30]. Further work is needed to determine the mechanism(s) by which both postactivation depression and reflex activation history are modulated.

Intuitively, it makes sense that a presynaptic control is involved in quadriceps AMI. The presence of the joint effusion should result in a great deal of afferent traffic traveling towards the central nervous system. The ability to gate this overflow of afferent information would allow supraspinal mechanisms to take over and regulate movement at the knee. Supraspinal control of the musculature crossing the knee joint may be preferred, as it would permit efficient and precise control of movement, possibly preventing further unwanted motion at the joint. Further data are needed to determine the potential role of supraspinal systems in quadriceps AMI. Additionally, data examining the involvement of spinal and supraspinal pathways in the presence of joint injury or effusion should be obtained during functional movements, to see if neural control mechanisms are similar at rest and during activity.

Plasma catecholamine levels did not change following the introduction of the needle. We felt that the injection could have been viewed as a stressor by the volunteers, activating the sympathetic nervous system and hypothalamic-pituitary adrenal axis, resulting in increased levels of catecholamines in the blood [2]. An increased sympathetic response has been suggested as resulting in Hmax and Mmax facilitation [8, 20, 22]. If a sympathetic response did indeed occur, resulting in a facilitation of 
the Hmax or Mmax, it may blunt the inhibitory response and cause us to underestimate the amount of inhibition that occurs with an effusion. The fact that plasma catecholamine levels did not increase suggests that stress due to the injection did not affect the measurements.

We conclude that quadriceps AMI elicited by an experimental knee joint effusion is in part regulated by a presynaptic spinal mechanism. Future work should focus on whether presynaptic mechanisms are activated in patients following joint damage.

Acknowledgements This work was supported in part by a grant from the National Institutes of Health to the University of Virginia General Clinical Research Center, number M01RR00847. Additional support was provided by the University of Virginia School of Medicine. The experiments comply with the current laws of the country in which they were performed.

\section{References}

1. Baldissera F, Hultborn $\mathrm{H}$, Illert $\mathrm{M}$ (1981) Integration in spinal neuronal systems. In: Brooks V (ed) Handbook of physiology. American Physiological Society, Bethesda, pp 509-596

2. Chrousos GP, Gold PW (1992) The concepts of stress and stress system disorders. Overview of physical and behavioral homeostasis. JAMA 267:1244-1252

3. Curtis DR, Eccles JC (1960) Synaptic action during and after repetitive stimulation. J Physiol Lond 150:374-398

4. Eccles JC (1964) Presynaptic inhibition in the spinal cord. Prog Brain Res 12:65-91

5. Ferrell WR (1987) The effect of acute joint distension on mechanoreceptor discharge in the knee of the cat. Q J Exp Physiol 72:493-499

6. Hopkins JT, Ingersoll CD (2000) Arthrogenic muscle inhibition: a limiting factor in joint rehabiliation. J Sport Rehab 9:135-159

7. Hopkins JT, Ingersoll CD, Cordova ML, Edwards JE (2000) Intrasession and intersession reliability of the soleus H-reflex in supine and standing positions. Electromyogr Clin Neurophysiol 40:89-94

8. Hopkins JT, Ingersoll CD, Edwards JE, Klootwyk TE (2002) Cryotherapy and TENS decrease arthrogenic muscle inhibition of the vastus medialis following knee joint effusion. J Athl Train 37:25-32

9. Hopkins JT, Ingersoll CD, Krause BA, Edwards JE, Cordova ML (2001) Effect of knee joint effusion on quadriceps and soleus motoneuron pool excitability. Med Sci Sports Exerc 33:123-126

10. Hopkins JT, Wagie NC (2003) Intrasession and intersession reliability of the quadriceps Hoffmann reflex. Electromyogr Clin Neurophysiol 43:85-89

11. Hurley MV (1997) The effects of joint damage on muscle function, proprioception, and rehabilitation. Man Ther 2:11-17
12. Hurley MV, Jones DW, Newham DJ (1994) Arthrogenic quadriceps inhibition and rehabilitation of patients with extensive traumatic knee injuries. Clin Sci (Colch) 86:305-310

13. Hurley MV, Jones DW, Wilson D, Newham DJ (1992) Rehabilitation of quadriceps inhibited to isolated rupture of the anterior cruciate ligament. $\mathbf{J}$ Orthop Rheumatol 5:145-154

14. Hurley MV, Newham DJ (1993) The influence of arthrogenous muscle inhibition on quadriceps rehabilitation of patients with early, unilateral osteoarthritic knees. Br J Rheumatol 32:127131

15. Hurley MV, Scott DL, Rees J, Newham DJ (1997) Sensorimotor changes and functional performance in patients with knee osteoarthritis. Ann Rheum Dis 56:641-648

16. Iles JF, Stokes M, Young A (1990) Reflex actions of knee joint afferents during contraction of the human quadriceps. Clin Physiol 10:489-500

17. Jackson DW, Evans NA, Thomas BM (2002) Accuracy of needle placement into the intra-articular space of the knee. J Bone Joint Surg Am 84:15221527

18. Jankowska $E$ (1981) Interneurons in the spinal cord. Trends Neurosci 4:230-233

19. Meunier S, Pierrot-Deseilligny E (1998) Cortical control of presynaptic inhibition of Ia afferents in humans. Exp Brain Res 119:415-426

20. Palmieri RM, Ingersoll CD, Edwards JE, Hoffman MA, Stone MB, Babington JE, Cordova ML, Krause BA (2003) Arthrogenic muscle inhibition is not present in the limb contralateral to a simulated knee joint effusion. Am $\mathbf{J}$ Phys Med Rehabil 82:910-916

21. Panizza M, Nilsson J, Roth BJ, Basser PJ, Hallett M (1992) Relevance of stimulus duration for activation of motor and sensory fibers: implications for the study of H-reflexes and magnetic stimulation. Electroencephalogr Clin Neurophysiol 85:22-29
22. Petrik J, Amendola A, Rampersand R, Mabey S, Hayes KC (1996) The effects of an isolated ankle effusion on H-reflex amplitude, viscoelasticity, and postural control of the ankle. American Orthopedic Society of Sports Medicine, Rosemont, IL

23. Pierrot-Deseilligny E, Mazevet D (2000) The monosynaptic reflex: a tool to investigate motor control in humans. Interest and limits. Neurophysiol Clin 30:67-80

24. Shakespeare DT (1985) Reflex inhibition of the quadriceps after meniscectomy: lack of association with pain. Clin Physiol 5:137-144

25. Spencer JD, Hayes KC, Alexander IJ (1984) Knee joint effusion and quadriceps reflex inhibition in man. Arch Phys Med Rehabil 65:171-177

26. Stokes M, Young A (1984) The contribution of reflex inhibition to arthrogenous muscle weakness. Clin Sci (Colch) 67:7-14

27. Suter E, Herzog W (2000) Does muscle inhibition after knee injury increase the risk of osteoarthritis? Exerc Sport Sci Rev 28:15-18

28. Thompson FJ, Parmer R, Reier PJ (1998) Alteration in rate modulation of reflexes to lumbar motoneurons after midthoracic spinal cord injury in the rat. I. Contusion injury. J Neurotrauma $15: 495-508$

29. Trimble MH, Du P, Brunt D, Thompson FJ (2000) Modulation of triceps surae $\mathrm{H}$-reflexes as a function of the reflex activation history during standing and stepping. Brain Res 858:274-283

30. Voigt M, Sinkjaer T (1998) The H-reflex in the passive human soleus muscle is modulated faster than predicted from post-activation depression. Brain Res 783:332-346 